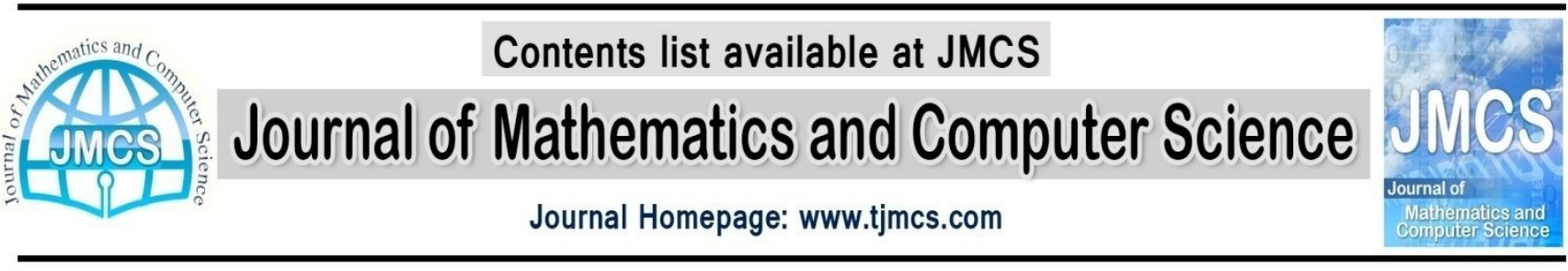

\title{
Two Efficient Approaches based on Radial Basis Functions to Nonlinear Time-dependent Partial Differential Equations
}

\author{
Jafar Biazar, M ohammad Hosami \\ Department of Applied M athematics, Faculty of Mathematical Sciences, University of Guilan, Rasht, Iran \\ biazar@guilan.ac.ir,mhosami@phd.guilan.ac.ir
}

Article history:

Received August 2013

Accepted October 2013

Available online October 2013

\begin{abstract}
In this paper, two numerical meshless approaches, based on radial basis functions (RBFs) are applied to solve nonlinear time-dependent partial differential equations. Both of these approaches are based on Kansa meshless method. In these procedures the time is descritized to small time steps, the space is also descritized in each sub-domain. Then by the Kansa collocation method, by using RBFs, the approximate solution, in each step, is obtained. In the first approach, the nonlinear terms are eliminated, by linearization. In the second approach nonlinear equations are solved directly, by Fix point method. Four examples are provided to illustrate the efficiency and the reliability of these approaches. The results are also compared with each other.
\end{abstract}

Keywords: M eshless M ethods; Radial Basis functions; Time-dependent nonlinear partial differential equations

\section{Introduction}

During the last decade, meshless methods have received much attention. Due to the difficulty of the mesh generation problem, meshless methods for simulation of the numerical problems are employed. Meshless methods mainly contain GFEM [1], EFGM [2], and meshless methods using radial basis functions (RBFs) [3]. Radial basis functions (RBFs) interpolation is a technique for representing a function starting with data on scattered points. The RBFs can be of various types, such as: polynomials of a given degree; linear, quadratic, cubic, etc; thin plate spline (TPS), multiquadrics (MQ), inverse multiquadrics (IMQ), Gaussian forms (GA), etc. In the cases of inverse quadratic, inverse multiquadric (IMQ), and Gaussian (GA), the matrix of coefficients of RBFs interpolation is positive definite, and 
for multiquadric (MQ) it has one positive eigenvalue and the remaining ones are all negative [4]. For more details, interested readers are referred to [5]. In 1990, Kansa proposed a scheme for estimating the solution of differential equations, using RBFs. Recently, Kansa's method was modified to solve various ordinary and partial differential equations including nonlinear time-dependent partial differential equations [7-11]. There are some common methods for time-dependent nonlinear partial differential equations such as Homotopy perturbation method, Variational interation method, and etc [12-13]. Recently some methods based on method of lines have been used to solve these types of differential equations [14-17]. Combination of these methods and RBF methods are also applied to solve time-dependent nonlinear partial differential equations. In these methods the time is descritized to small time steps, and space is also descritized in each sub-domain, and then by the collocation approaches using RBFs the approximate solution is obtained, in each step.

In this paper, two approaches for this method are introduced. To show the efficiency of the approaches, the methods are applied for solving two famous equations named Kuramoto-Sivashinsky and FisherBurger equations. In Section 2, the properties of radial basis functions are described. In Section 3, two approaches are introduced to solve the time-dependent nonlinear partial differential equations. These approaches are applied to solve Kuramoto-Sivashinsky, and Burger-Fisher equation. In Section 4, the numerical implementations of the methods are investigated. Conclusions and discussions are appeared in the final section.

\section{Radial basis functions}

Let $\varphi: \mathbb{R}^{+} \rightarrow \mathbb{R}$ be a continuous function with $\varphi(0) \geq 0$. A radial basis function on $\mathbb{R}^{d}$ is a function of the following form

$$
\varphi\left(\left\|X-X_{i}\right\|\right)
$$

where $X, X_{i} \in \mathbb{R}^{d}$, and $\|\cdot\|$ denotes the Euclidean norm. If $\left\{X_{i}\right\}_{i=1}^{N}$ is a sequence of distinct points in $\mathbb{R}^{d}$, then, as usual, the approximation has the general following form

$$
S(X)=\sum_{i=1}^{N} \lambda_{i} \varphi\left(\left\|X-X_{i}\right\|\right), \quad \lambda_{i} \in \mathbb{R} .
$$

Most important properties of RBFs are; radial symmetry, smoothness, and certain properties of their Fourier transform. Some of the most important radial basis functions are shown in Table1.

\subsection{Using RBFs to approximate a function (Kansa method)}

Let us choose $N$ distinct nodes $\left(X_{j}, j=1,2, \ldots, N\right)$ in the interval $[a, b]$. In RBF methods, a function $u(x)$ is approximated by $u^{*}(x)$ as

$$
u^{*}(x)=\sum_{j=1}^{N} \lambda_{j} \varphi\left(r_{j}\right)=\Phi^{T}(r) \lambda
$$


Table1: Some of the most important radial basis functions

\begin{tabular}{l|c}
\hline$r=\left\|X-X_{i}\right\|, \quad c>0$. & \\
\hline RBF & Definition \\
\hline Multiquadric (MQ) & $\sqrt{r^{2}+c^{2}}$ \\
Inverse Multiquadric (IMQ) & $1 / \sqrt{r^{2}+c^{2}}$ \\
Thin-plate Spline (TPS) & $r^{2} \log r$ \\
Guassian (GA) & $\exp \left(-r^{2} / c^{2}\right)$ \\
Inverse Quadric & $1 /\left(r^{2}+c^{2}\right)$ \\
\hline
\end{tabular}

where $r_{j}=\left\|X-X_{j}\right\|, \lambda_{j}$ s are time dependent unknowns to be determined. $\varphi(r), r \geq 0$ is some RBF. Also

$$
\Phi(r)=\left[\varphi_{1}(r), \varphi_{2}(r), \ldots, \varphi_{N}(r)\right]^{T},
$$

and

$$
\lambda=\left[\lambda_{1}, \lambda_{2}, \ldots, \lambda_{N}\right]^{T}
$$

By considering $u^{*}\left(X_{i}\right)=u_{i}$, Eq. (1) can be represented as the following

$$
A \lambda=u
$$

where $u=\left[u_{1}, u_{2}, \ldots, u_{N}\right]^{T}$, and

$$
A=\left[\begin{array}{l}
\Phi^{T}\left(r_{1}\right) \\
\Phi^{T}\left(r_{2}\right) \\
\vdots \\
\Phi^{T}\left(r_{N}\right)
\end{array}\right]=\left[\begin{array}{cccc}
\varphi_{1}\left(r_{1}\right) & \varphi_{2}\left(r_{1}\right) & \ldots & \varphi_{N}\left(r_{1}\right) \\
\varphi_{1}\left(r_{2}\right) & \varphi_{2}\left(r_{2}\right) & \ldots & \varphi_{N}\left(r_{2}\right) \\
\vdots & \vdots & \ddots & \vdots \\
\varphi_{1}\left(r_{N}\right) & \varphi_{2}\left(r_{N}\right) & \ldots & \varphi_{N}\left(r_{N}\right)
\end{array}\right]
$$

From Equations (1) and (2), $u^{*}(x)$ can be written as;

$$
u^{*}(x)=\Phi^{T}(r) A^{-1} u \text {. }
$$




\section{Using RBFs for time dependent PDE}

To illustrate the method, it is used to solve the Kuramoto-Sivashinsky equation. The general form of this equation is as follows

$$
u_{t}+u u_{x}+\alpha u_{x x}+\sigma u_{x x x}+\beta u_{x x x x}=0
$$

Two approaches based on the meshless method, using RBFs, are introduced to solve this equation.

\subsection{First approach}

Consider Eq. (4) with the following boundary and initial conditions;

$$
\begin{array}{ll}
u(a, t)=g_{1}(t), & u(b, t)=g_{2}(t), \quad t>0, \\
u(x, 0)=f(x), & a \leq x \leq b .
\end{array}
$$

By applying the Crank-Nicolson scheme to Eq. (4), the following difference equation is obtained.

$$
\begin{aligned}
& {\left[\frac{u^{n+1}-u^{n}}{\Delta t}\right]+\left[\frac{\left(u u_{x}\right)^{n+1}+\left(u u_{x}\right)^{n}}{2}\right]+\alpha\left[\frac{\left(u_{x x}\right)^{n+1}+\left(u_{x x}\right)^{n}}{2}\right]+\sigma\left[\frac{\left(u_{x x x}\right)^{n+1}+\left(u_{x x x}\right)^{n}}{2}\right]} \\
& +\beta\left[\frac{\left(u_{x x x x}\right)^{n+1}+\left(u_{x x x x}\right)^{n}}{2}\right]=0
\end{aligned}
$$

where $u^{n+1}=u\left(t^{n+1}\right), t^{n+1}=t^{n}+\Delta t$, and $\Delta t$ is the time step. The main idea in this approach is linearization of the nonlinear term. To linearize the nonlinear term $u u_{x}$, one can use following formula

$$
\left(u u_{x}\right)^{n+1}=u^{n} u_{x}^{n+1}+u^{n+1} u_{x}^{n}-u^{n} u_{x}^{n}
$$

Results of substitution (8) into (7) follows

$$
\left[\frac{u^{n+1}-u^{n}}{\Delta t}\right]+\left[\frac{u^{n} u_{x}^{n+1}+u^{n+1} u_{x}^{n}}{2}\right]+\alpha\left[\frac{u_{x x}^{n+1}+u_{x x}{ }^{n}}{2}\right]+\sigma\left[\frac{u_{x x x}{ }^{n+1}+u_{x x x}{ }^{n}}{2}\right]+\beta\left[\frac{u_{x x x x}{ }^{n+1}+u_{x x x x}{ }^{n}}{2}\right]=0 .
$$

Terms, can be rearranged as follows

$$
u^{n+1}+\frac{\Delta t}{2}\left[u^{n} u_{x}^{n+1}+u^{n+1} u_{x}^{n}+\alpha u_{x x}{ }^{n+1}+\sigma u_{x x x}{ }^{n+1}+\beta u_{x x x x}{ }^{n+1}\right]=u^{n}-\frac{\Delta t}{2}\left[\alpha u_{x x}{ }^{n}+\sigma u_{x x x}{ }^{n}+\beta u_{x x x x}{ }^{n}\right],
$$

where $u^{n}$ represents the $n$ 'th iteration of the approximate solution. Suppose that approximate solution of Eq. (9) has the following form 


$$
u^{n}\left(x_{i}\right)=\sum_{j=0}^{N} \lambda_{j}^{n} \varphi\left(r_{i j}\right)
$$

where $x_{i}=a+i \Delta x$ are collocation points in $[a, b], r_{i j}=\left\|x_{i}-x_{j}\right\|, \lambda_{j}{ }^{n}$ are unknown values to be determined, and $\varphi(r)$ is a radial basis function. By substituting (10) in to (9), the following equation will be obtained, for all interior points $x_{i}(i=1,2 . ., N-1)$.

$$
\begin{aligned}
& \sum_{j=0}^{N} \lambda_{j}{ }^{n+1} \varphi\left(r_{i j}\right)+\frac{\Delta t}{2}\left[u^{n} \times \sum_{j=0}^{N} \lambda_{j}^{n+1} \varphi_{x}\left(r_{i j}\right)+u_{x}{ }^{n} \times \sum_{j=0}^{N} \lambda_{j}{ }^{n+1} \varphi\left(r_{i j}\right)+\alpha \sum_{j=0}^{N} \lambda_{j}{ }^{n+1} \varphi_{x x}\left(r_{i j}\right)+\sigma \sum_{j=0}^{N} \lambda_{j}{ }^{n+1} \varphi_{x x x}\left(r_{i j}\right)\right. \\
& \left.+\beta \sum_{j=0}^{N} \lambda_{j}^{n+1} \varphi_{x x x x}\left(r_{i j}\right)\right]=u^{n}-\frac{\Delta t}{2}\left[\alpha u_{x x}{ }^{n}+\sigma{u_{x x x}}^{n}+\beta u_{x x x x}{ }^{n}\right] .
\end{aligned}
$$

The boundary conditions (5) and (6) take the following form

$$
\sum_{j=0}^{N} \lambda_{j}^{n+1} \varphi\left(r_{1 j}\right)=g_{1}(t), \quad \sum_{j=0}^{N} \lambda_{j}^{n+1} \varphi\left(r_{N j}\right)=g_{2}(t) .
$$

At each step, $\lambda_{j}{ }^{n}$ s are known and the system (11) and (12) contains $N+1$ unknowns $\lambda_{j}{ }^{n+1}$, which can be easily determined by some methods such as Gaussian elimination, QR factorization, and etc. By solving this system of linear equations, $\lambda_{j}^{n+1} \mathrm{~s}$, are determined and from (10)

$$
u^{n+1}(x)=\sum_{j=0}^{N} \lambda_{j}^{n+1} \varphi\left(r_{j}\right), \quad r_{j}=\left\|x-x_{j}\right\| .
$$

\subsection{Second approach}

In this approach the domain descritize in directions, time and space. Using radial basis functions to approximate the solution of the equation in each sub-domain, a closed form of the solution is resulted, and this is used to initial condition of the next sub-domain. In this approach the equation (4) is solved in each subdomain using the collocation technique. The system of nonlinear equations which arises from nonlinear term is solved directly, without any linearization. This system can be solved via Fix point method. By the first approach notations in each subdomain, the following equations is obtained

$$
\begin{aligned}
& u_{t}^{n+1}+u^{n+1} u_{x}^{n+1}+\alpha u_{x x}{ }^{n+1}+\sigma u_{x x x}{ }^{n+1}+\beta u_{x x x x}{ }^{n+1}=0, \\
& u^{n+1}(a, t)=g_{1}(t), \quad u(b, t)=g_{2}(t), \quad t>0, \\
& u^{n+1}(x, 0)=u^{n}(x), \quad a \leq x \leq b \text {. }
\end{aligned}
$$

By substituting (10) into (13), the following equation will be obtained 


$$
\begin{aligned}
& \sum_{j=0}^{N} \lambda_{j}^{n+1} \varphi_{t}\left(r_{i j}\right)+\sum_{j=0}^{N} \lambda_{j}^{n+1} \varphi\left(r_{i j}\right) \times \sum_{j=0}^{N} \lambda_{j}{ }^{n+1} \varphi_{x}\left(r_{i j}\right) \\
& \left.+\alpha \sum_{j=0}^{N} \lambda_{j}^{n+1} \varphi_{x x}\left(r_{i j}\right)+\sigma \sum_{j=0}^{N} \lambda_{j}{ }^{n+1} \varphi_{x x x}\left(r_{i j}\right)+\beta \sum_{j=0}^{N} \lambda_{j}{ }^{n+1} \varphi_{x x x x}\left(r_{i j}\right)\right]=0,
\end{aligned}
$$

for all interior points $x_{i}(i=1,2 . ., N-1)$. The system (14) contains $N-1$ nonlinear equations. With the equations arise from boundary and initial conditions; a system of $N+1$ nonlinear equations with $N+1$ unknowns $\lambda_{j}^{n+1}$, are obtained. This nonlinear system can be solved by some methods such as, Fix point, Newton method, and etc.

\section{Numerical implementation}

Two approaches are applied to solve two famous Equations to comparison the numerical solutions. First, the numerical solution of Kuramoto-Sivashinsky equation is considered. Additionally, numerical solution of Burger-Fisher Equation is obtained by two approaches.

\subsection{Kuramoto-Sivashinsky equation}

Example 1: Consider the equation (4), with $\alpha=1, \sigma=4$, and $\beta=1$.

$$
u_{t}+u u_{x}+u_{x x}+4 u_{x x x}+u_{x x x x}=0
$$

with initial and boundary conditions

$$
\begin{aligned}
& u(x, 0)=C+9-15\left[\tanh \left(k\left(x-x_{0}\right)\right)+\tanh ^{2}\left(k\left(x-x_{0}\right)\right)-\tanh ^{3}\left(k\left(x-x_{0}\right)\right)\right], \\
& u(a, t)=C+9-15\left[\tanh \left(k\left(a-C t-x_{0}\right)\right)+\tanh ^{2}\left(k\left(a-C t-x_{0}\right)\right)-\tanh ^{3}\left(k\left(a-C t-x_{0}\right)\right)\right], \\
& u(b, t)=C+9-15\left[\tanh \left(k\left(b-C t-x_{0}\right)\right)+\tanh ^{2}\left(k\left(b-C t-x_{0}\right)\right)-\tanh ^{3}\left(k\left(b-C t-x_{0}\right)\right)\right] .
\end{aligned}
$$

The exact solution is

$$
u(x, t)=C+9-15\left[\tanh \left(k\left(x-C t-x_{0}\right)\right)+\tanh ^{2}\left(k\left(x-C t-x_{0}\right)\right)-\tanh ^{3}\left(k\left(x-C t-x_{0}\right)\right)\right] .
$$

This equation has been solved by two approaches, with $C=6, k=0.5$, and $x_{0}=-10$, in $[a, b]=[-20,10]$. In this paper multiquadric RBF (MQ) has been applied. In Fig. 1 the solitary wave propagation for the Kuramoto-Sivashinsky equation (15) is shown. Time step in first and second approach is 0.01 . It is obvious that the first approach has a good approximate solution as well as the second approach. To compare the approximate solutions with the exact one, the RMS error in some non-collocation points at different times are presented in Table 2.

$$
R M S=\sqrt{\frac{1}{N} \sum_{i=1}^{N}\left|u_{\text {exact }}\left(x_{i}, t\right)-u_{\text {approximate }}\left(x_{i}, t\right)\right|^{2}}
$$



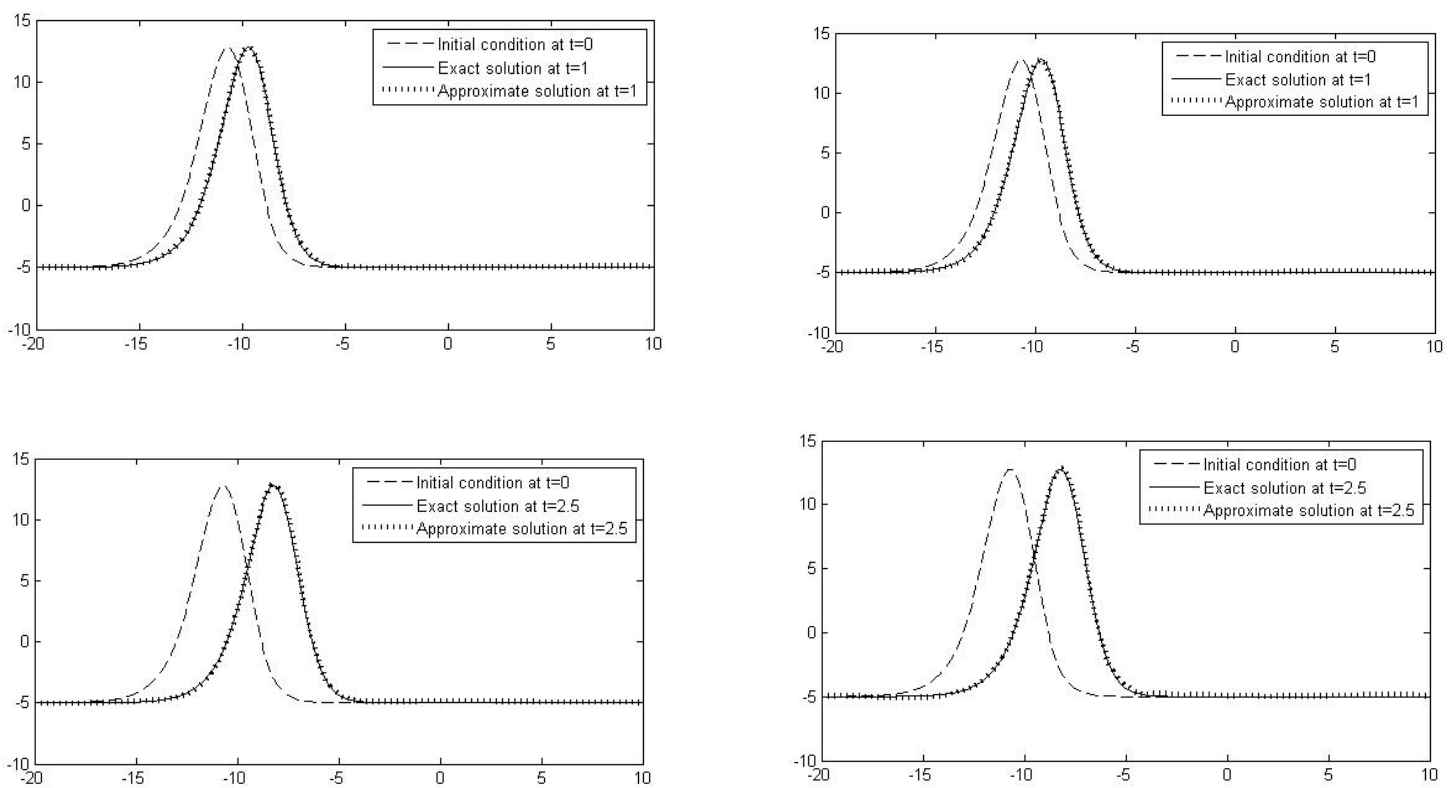

Fig. 1: The initial, exact, and approximate solutions of Equation (15), at different times by the first (left), and the second approach (right)

Table 2: The values of RMS error of approximate solution of example (1) at different values of $t$

\begin{tabular}{c|c|c|c|c|c|c}
\hline \multicolumn{2}{c}{ time } & 0.5 & 1 & 1.5 & 2 & 2.5 \\
\hline \multirow{2}{*}{ RMS } & First approach & $3.11 \mathrm{e}-2$ & $6.66 \mathrm{e}-2$ & $7.44 \mathrm{e}-2$ & $9.24 \mathrm{e}-2$ & $1.15 \mathrm{e}-1$ \\
\cline { 2 - 7 } & Second approach & $6.42 \mathrm{e}-2$ & $7.66 \mathrm{e}-2$ & $8.59 \mathrm{e}-2$ & $9.27 \mathrm{e}-2$ & $1.26 \mathrm{e}-1$ \\
\hline
\end{tabular}

Example 2: Consider the equation (4), with $\alpha=1, \sigma=1$, and $\beta=0$,

$$
u_{t}+u u_{x}+u_{x x}+u_{x x x}=0
$$

with initial and boundary conditions

$$
\begin{aligned}
& u(x, 0)=C+\frac{15}{19} \sqrt{\frac{11}{19}}\left[11 \tanh ^{3}\left(k\left(x-x_{0}\right)\right)-9 \tanh \left(k\left(x-x_{0}\right)\right)\right], \\
& u(a, t)=C+\frac{15}{19} \sqrt{\frac{11}{19}}\left[11 \tanh ^{3}\left(k\left(a-C t-x_{0}\right)\right)-9 \tanh \left(k\left(a-C t-x_{0}\right)\right)\right], \\
& u(b, t)=C+\frac{15}{19} \sqrt{\frac{11}{19}}\left[11 \tanh ^{3}\left(k\left(b-C t-x_{0}\right)\right)-9 \tanh \left(k\left(b-C t-x_{0}\right)\right)\right] .
\end{aligned}
$$


In Fig. 2 the solitary wave propagation for the Kuramoto-Sivashinsky equation (16) with $C=0.5, k=\frac{1}{2} \sqrt{\frac{11}{19}}$, and $x_{0}=-10$, in the interval $[a, b]=[-30,30]$ is given. Time step in first and second approaches is $\Delta t=0.1$. The values of RMS error at different values of $t$ are presented in Table 3.
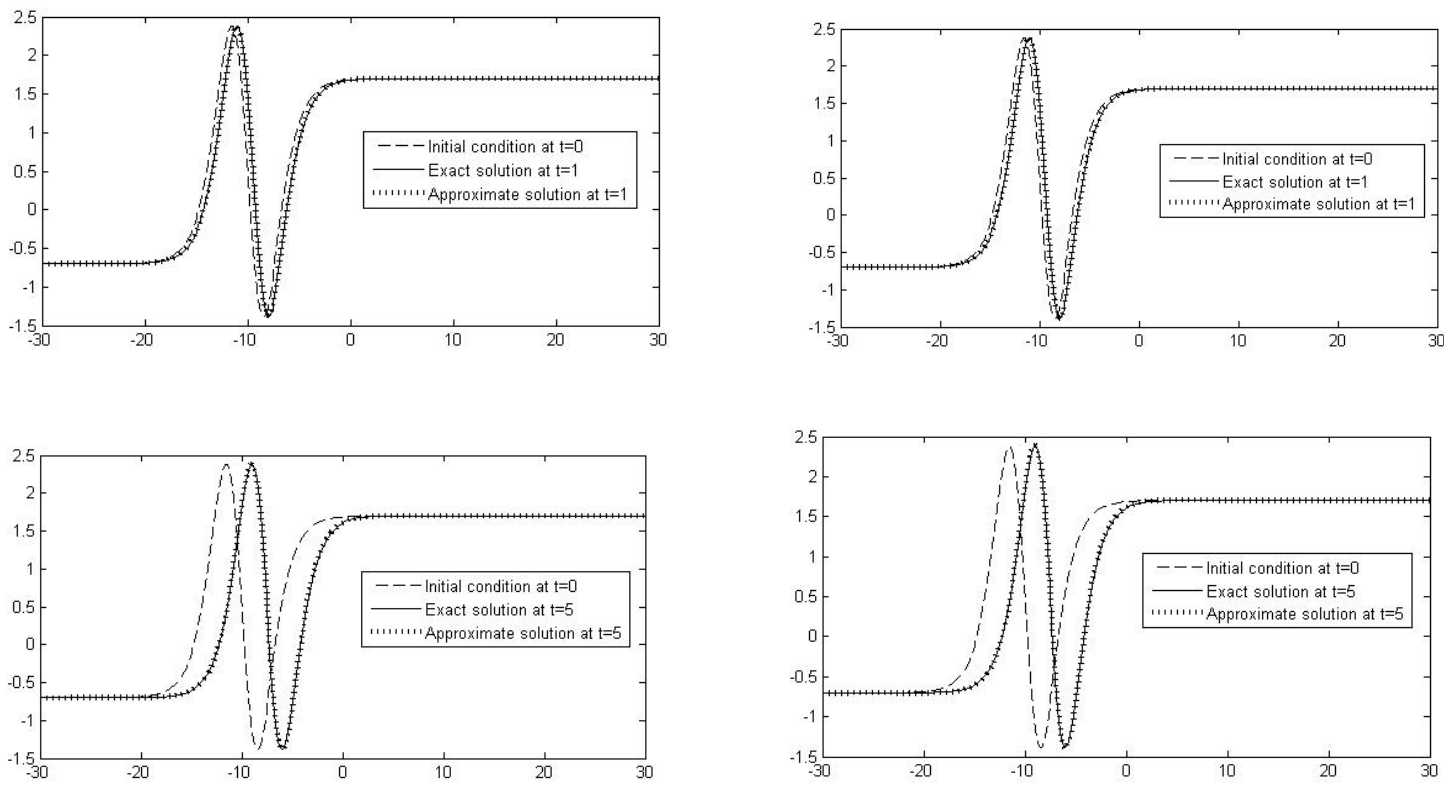

Fig. 2: The initial, exact, and approximate solutions of Equation (16), at different times by the first (left), and the second approach (right)

Table 3: The values of RMS error of approximate solution of example (2) at different values of $t$

\begin{tabular}{c|c|c|c|c|c|c}
\hline \multicolumn{2}{c}{ time } & 0.5 & 1 & 1.5 & \multicolumn{2}{c}{2} \\
\hline \multirow{2}{*}{ RMS } & First approach & $2.98 \mathrm{e}-4$ & $6.06 \mathrm{e}-4$ & $7.73 \mathrm{e}-4$ & $1.07 \mathrm{e}-3$ & $1.22 \mathrm{e}-3$ \\
\cline { 2 - 7 } & Second approach & $1.39 \mathrm{e}-3$ & $2.71 \mathrm{e}-3$ & $3.70 \mathrm{e}-3$ & $4.76 \mathrm{e}-3$ & $9.66 \mathrm{e}-3$ \\
\hline
\end{tabular}

\subsection{Burger-Fisher equation}

Consider the following equation

$$
u_{t}+\alpha u^{\sigma} u_{x}-u_{x x}=\beta u\left(1-u^{\sigma}\right)
$$

with the exact solution 


$$
u(x, t)=\left(1 / 2+1 / 2 \tanh \left(\frac{-\alpha \sigma}{2(\sigma+1)}\left(x-\left(\frac{\alpha}{\sigma+1}+\frac{\beta(\sigma+1)}{\alpha}\right) t\right)\right)\right)^{1 / \sigma} .
$$

This equation is general form of Burger-Fisher equation. By two approaches, the solution of this equation for various values of the parameters $\alpha, \beta$, and $\sigma$ is investigated.

Example 3: Consider the generalize Burgers-Fisher equation (1.1), with $\alpha=1, \beta=0$, and $\sigma=1$. The initial condition and boundary conditions are as follows

$$
\begin{aligned}
& u(x, 0)=1 / 2+1 / 2 \tanh \left(\frac{-x}{4}\right), \\
& u(a, t)=1 / 2+1 / 2 \tanh \left(\frac{-1}{4}\left(a-\left(\frac{1}{2}\right) t\right)\right), \quad u(b, t)=1 / 2+1 / 2 \tanh \left(\frac{-1}{4}\left(b-\left(\frac{1}{2}\right) t\right)\right) .
\end{aligned}
$$

Fig. 3 shows the approximate solution of this equation by first and second approaches at different times. The values of RMS error at various times are presented in Table 4. Time step in the first and second approaches is taken as $\Delta t=0.1$.
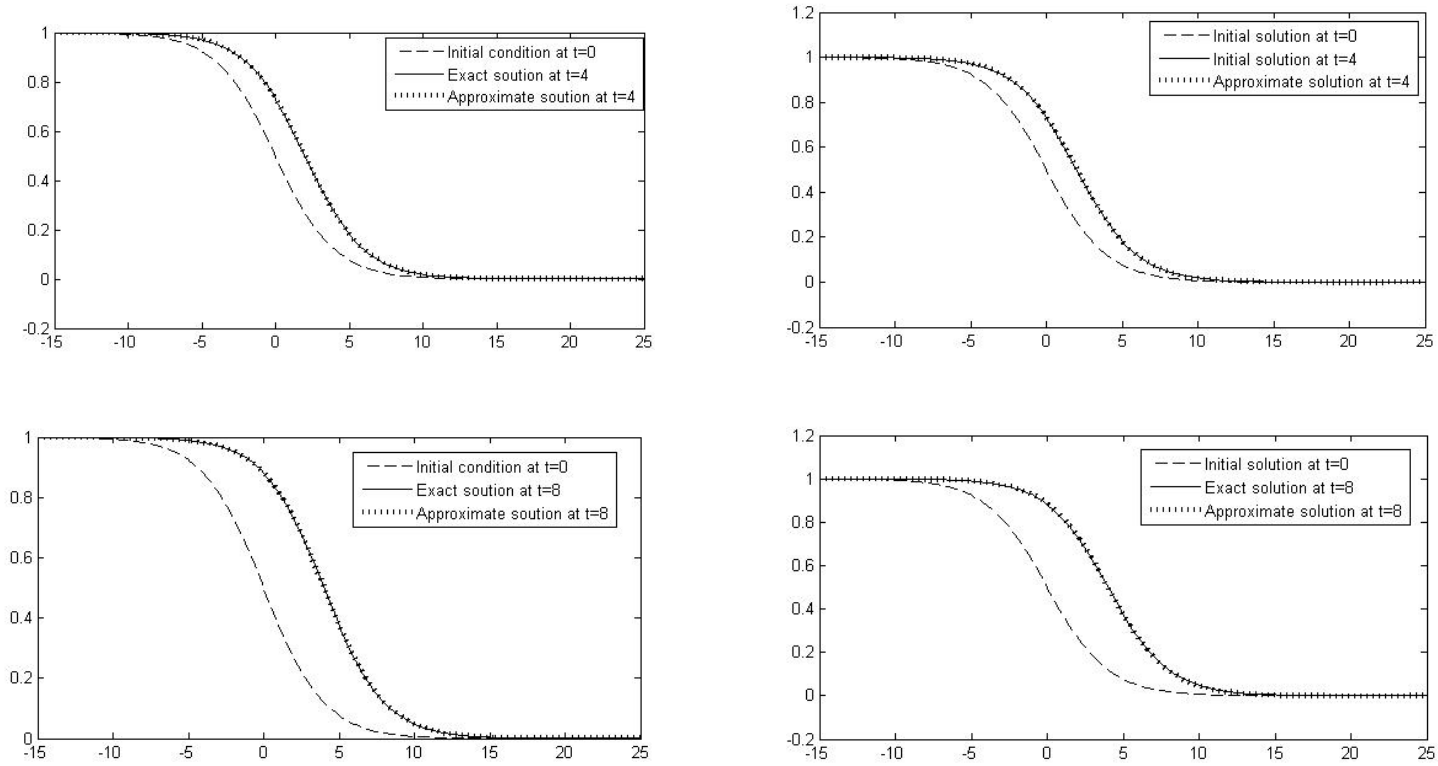

Fig. 3: The initial, exact, and approximate solutions of Equation (17), with $\alpha=1, \beta=0$, and $\sigma=1$ at various times by the first (left), and the second approach (right)

Table 4: The values of RMS error of approximate solution of example (3) at different values of $t$

\begin{tabular}{c|c|c|c|c|c|c}
\hline \multicolumn{2}{c}{ time } & 0.5 & 1 & 4 & 6 & 8 \\
\hline \multirow{2}{*}{ RMS } & First approach & $1.03 \mathrm{e}-4$ & $1.48 \mathrm{e}-4$ & $2.35 \mathrm{e}-4$ & $3.72 \mathrm{e}-4$ & $4.42 \mathrm{e}-4$ \\
\cline { 2 - 7 } & Second approach & $1.74 \mathrm{e}-4$ & $3.37 \mathrm{e}-4$ & $1.30 \mathrm{e}-3$ & $1.71 \mathrm{e}-3$ & $2.22 \mathrm{e}-3$ \\
\hline
\end{tabular}


Example 4: Consider the Burgers-Fisher equation (1.1), for $\alpha=2, \beta=1$, and $\sigma=1$. The initial condition and boundary conditions are as follows

$$
\begin{aligned}
& u(a, t)=1 / 2+1 / 2 \tanh \left(\frac{-x}{2}\right), \\
& u(a, t)=1 / 2+1 / 2 \tanh \left(\frac{-1}{2}(a-3 t)\right), \\
& u(b, t)=1 / 2+1 / 2 \tanh \left(\frac{-1}{2}(b-3 t)\right) .
\end{aligned}
$$

Fig. 4 shows the approximate solution of this equation by first and second approaches at various times. The values of RMS error at different values of times are presented in Table 5. Time step in first and second approaches is $\Delta t=0.01$.
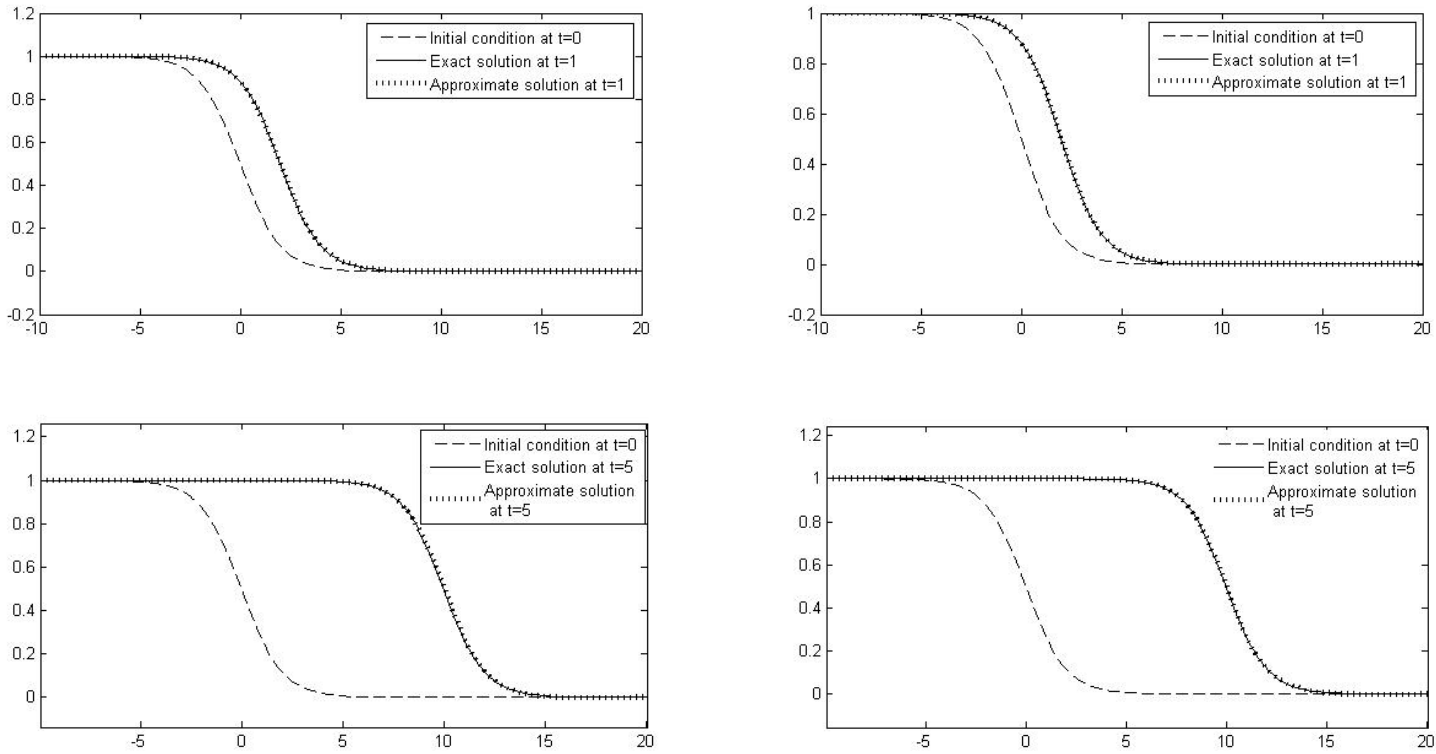

Fig. 4: The initial, exact, and approximate solutions of Equation (17), for $\alpha=2, \beta=1$, and $\sigma=1$, at various times by the first (left), and the second approach (right)

Table 5: The values of RMS error of approximate solution of example (4) at different values of $t$

\begin{tabular}{c|c|c|c|c|c|c}
\hline \multicolumn{2}{c}{ time } & 0.5 & 1 & 1.5 & 2 & 5 \\
\hline \multirow{2}{*}{ RMS } & First approach & $1.14 \mathrm{e}-4$ & $2.63 \mathrm{e}-4$ & $3.98 \mathrm{e}-4$ & $6.03 \mathrm{e}-4$ & $8.87 \mathrm{e}-4$ \\
\cline { 2 - 7 } & Second approach & $2.91 \mathrm{e}-4$ & $4.53 \mathrm{e}-4$ & $5.45 \mathrm{e}-4$ & $7.91 \mathrm{e}-4$ & $2.53 \mathrm{e}-3$ \\
\hline
\end{tabular}




\section{Conclusions}

Two approaches of meshless method using radial basis functions have been investigated. To show the efficiency of the approaches, they have been applied to solve two famous time-dependent nonlinear partial differential equations, such as Kuramoto-Sivashinsky, and Burger-Fisher equations. The results show, both the first and the second approaches are reliable and efficient to solve this time-dependent nonlinear PDE.

\section{References}

[1] Babuska I. Banerjee U. Osborn J.E. Survey of meshless and generalized finite element methods: A unified approach, in: Acta. Numer, Cambridge University Press, 2003, pp. 1-125.

[2] Belytschko T. Krongauze Y. Organ D. Fleming M. Krysl P. Meshless methods: An overview and recent developments, Comput. Methods. Appl. Math. 139 (1996) 3-47 (special issue on Meshless Methods).

[3] Kansa E.J. Multiquadrics-A scattered data approximation scheme with applications to computational fluid dynamics, II. Solution to parabolic, hyperbolic and elliptic partial differential equations, Comput. Math. Appl. 19 (1990) 147-161.

[4] Powell M. J. D. The theory of radial basis function approximation in 1990, Clarendon, 1992, Oxford.

[5] Liu G.R. Mesh Free Methods: Moving Beyond the Finite Element Method, CRC Press, 2003.

[6] Kansa E.J. Multiquadrics-A scattered data approximation scheme with applications to computational fluiddynamics-I surface approximations and partial derivative estimates. Comput. Math. Appl. 19 (1990) 127-145. [7] Dehghan M, Shokri A. Numerical solution of the nonlinear Klein-Gordon equation using radial basis functions. J. Comput. Appl. Math. 230 (2009) 400-410.

[8] Alipanah A, Dehghan M. Numerical solution of the nonlinear Fredholm integral equations by positive definite functions. Appl. Math. Comput. 190 (2007) 1754-1761.

[9] Sarra S. Adaptive radial basis function method for time dependent partial differential equations. Appl Numer Math 54 (2005) 79-94.

[10] Mai-Duy M, Tran-Cong T. An integrated-RBF technique based on Galerkin formulation for elliptic differential equations. Eng. Anal. Bound. Elem. 33 (2009) 191-199.

[11] Parand K, Abbasbandy S, Kazem S, Rezaei AR. An improved numerical method for a class of astrophysics problems based on radial basis functions. Phys. Scr. 83: (2011) 15011.

[12] Othman M. I. A. and Mahdy A. M. S. Differential Transformation Method and Variation Iteration Method for Cauchy Reaction-diffusion Problems, J. Math. Computer Sci. 1(2) (2010) 61-75.

[13] Ghazanfari B., Ghazanfari A.G., Fuladvand M., Modification of the Homotopy Perturbation Method for Numerical Solution of Nonlinear Wave and System of Nonlinear Wave Equations, J. Math. Computer Sci. 3 (2011) 212-224.

[14] Sharan M, Kansa EJ, Gupta S. Application of the multiquadric method for numerical solution of elliptic partial differential equations. Appl. Math. Comput. 84 (1997) 275-302.

[15] Zerroukat HPM, Chen C. A numerical method for heat transfer problems using collocation and radial basis functions. Int. J. Numer. Math. Eng. 42 (1998) 1263-1278.

[16] Mai-Duy N, Tran-Cong T. Numerical solution of differential equations using multiquadric radial basis function networks. Neural Networks 14 (2001) 185-199.

[17] Tatari M, Dehghan M. A method for solving partial differential equations via radial basis functions: Application to the heat equation. Eng. Anal. Bound. Elem. 34 (2010) 206-212. 